\title{
Effects of Concentrated Growth Factor and Nanofat on Aging Skin of Nude Mice Induced by D-Galactose
}

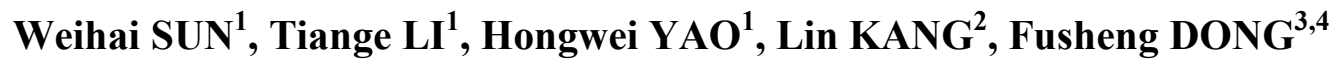 \\ ${ }^{1}$ Department of Esthetic Surgery, Beijing Evercare Medical Beauty Hospital, Beijing, China, \\ ${ }^{2}$ Department of Pathology, Hebei General Hospital, Hebei, China, ${ }^{3}$ Department of Oral and \\ Maxillofacial Surgery, Stomatology Hospital of Hebei Medical University, Shijiazhuang, Hebei, \\ China, ${ }^{4}$ Hebei Key Laboratory of Oral Medicine, Shijiazhuang, Hebei, China
}

Received January 7, 2021

Accepted March 23, 2021

Epub Ahead of Print May 12, 2021

\section{Summary}

This investigation studied the effect of concentrated growth factor and nanofat on aging skin of nude mice induced by D-galactose. BALB/C mice were randomly divided into five groups: 5 mice in the control group were fed normally without any intervention, 9 mice were treated with concentrated growth factor (CGF), 9 mice were treated with nanofat (NF), 9 mice were treated with $\mathrm{CGF}+\mathrm{NF}$, and 9 mice in the model group (no treatment after subcutaneous injection of D-galactose). Relevant indicators are measured and recorded. In skin and serum, SOD and GSH content in the model group were significantly lower than those in other groups $(P<0.05)$, and the MDA of the three treatment groups was significantly lower than that of the model group $(\mathrm{P}<0.05)$. Compared with the control group, the contents of total collagen, type I collagen and type III collagen in the NF group and model group were decreased in different degrees $(P<0.05)$; the contents of elastin and elastic fiber in the skin of nude mice in the model group and NF group were significantly decreased. Compared with the model group, he number of CD31 and VEGF in the treatment group was significantly increased $(P<0.01)$; the skin AGE content of three treatment groups was significantly lower $(P<0.05)$. These findings suggest that concentrated growth factor and nanofat may have a significant effect on delaying aging skin induced by D-galactose in nude mice.

\section{Key words}

Concentrated growth factor • Nanofat • Aging skin • Nude mice • D-galactose

\section{Corresponding author}

F. Dong, Department of Oral and Maxillofacial Surgery, Stomatology Hospital of Hebei Medical University, Shijiazhuang, Hebei 050017, China, Hebei Key Laboratory of Oral Medicine, Shijiazhuang, Hebei 050017, China, No. 383, Zhongshan East Road, Chang'an District, Shijiazhuang, Hebei 050017, China. Fax: 031186266796. E-mail: 954683986@qq.com

\section{Introduction}

Skin aging is a phenomenon of skin caused by a variety of factors. Due to the reduction of important components in the skin such as moisture, hyaluronic acid, elastin, and collagen, the skin shows reduced elasticity and softness, wrinkles, dryness, hyperkeratosis, and hyperpigmentation. With the development of society, anti-aging treatment of skin has become a focus of medical cosmetology. Glycosylation is a non-enzymatic reaction between the free amino acids in collagen, and it is also an oxidation reaction induced by oxygen-activated genes. Studies have shown that glycosylation induced by the reaction between reducing sugars and proteins is an important factor in causing body aging (Ha et al. 2004). Free radicals have a super oxidizing ability that can oxidize the unsaturated lipids of biological membranes and produce lipid peroxides (Liu et al. 2014). Malondialdehyde (MDA) is the final product of free radicals. Studies have shown that MDA can affect the exchange of substances between cells and ultimately lead to cell rupture and death (Herrling et al. 2008). The

PHYSIOLOGICAL RESEARCH • ISSN 1802-9973 (online) 
metabolic processes of various substances in the body can lead to the peroxidation reaction of free radicals, thereby causing the imbalance of free radicals. Once the damage caused by free radicals exceeds the body's self-recovery ability, it will lead to changes in the differentiation state of cells, affect the differentiation ability of cells, and, ultimately, lead to skin aging.

The fibrin gel in concentrated growth factors (CGFs) is a three-dimensional network structure formed by fibrin of different sizes in which a large number of white blood cells are embedded, indicating that it has a good anti-infection and anti-inflammatory ability. There are a large number of CD34+ cells and a large amount of vascular endothelial growth factor, fibroblast growth factor, and other active substances in CGFs, which play an important role in immune regulation and angiogenesis (Osawa et al. 1996).

Adipose stem cells (ASCs) can differentiate into vascular endothelial cells and adipocytes and can promote the formation of blood vessels and adipose tissue. They are widely present in the human body, and to a certain extent, they can be applied to the filling of soft tissues (Deng et al. 2014). The nanofat prepared by mechanical emulsification is small, without additives, and rich in ASCs. They can be injected subcutaneously or intradermally through fine needles to have anti-aging effects.

In recent years, the application of CGF in medical cosmetology has also achieved certain results, but the efficacy of CGF combined with nanofat has not been reported. In this study, BALB/c mice were used as the experimental object to explore the therapeutic effects of CGF, nanofat, and their mixture on a D-galactoseinduced skin aging model to explore the anti-aging mechanism and provide a new treatment method for clinical anti-aging therapy.

\section{Methods}

\section{Experimental animals}

A total of $41 \mathrm{SPF}$ BALB/c nude mice, male, weighing 15.1-16.7 g (SPF [Beijing] Biotechnology Co., Ltd.) were selected. The experiment was started after one week of rearing in a barrier environment. The 41 nude mice were randomly divided into five groups: 5 animals were in the normal control group (fed normally without any intervention), 9 animals were in the concentrated growth factor (CGF) group, 9 animals were in the nanofat (NF) group, 9 animals were in the combined treatment group (CGF+NF), and 9 animals were in the model group (no treatment after subcutaneous injection of D-galactose). According to the Laboratory Animal Guide, they were fed under the conditions of $20-25^{\circ} \mathrm{C}$ (daily temperature difference $\leq 3{ }^{\circ} \mathrm{C}$ ), relative humidity of $40-60 \%$ and light alternating for $12 \mathrm{~h}$, Mice could drink filtered water and standard pellet rat food at will during culture. The certificate number of the laboratory facility of the animal breeding room was SYXK (Beijing) 2002-0016. The laboratory animal use license number was SYXK (Beijing) 2014-0003.

\section{Experimental reagents and instruments}

Main reagent: D-galactose (Sinopharm Chemical Reagent Co., Ltd.); $0.9 \%$ sodium chloride injection (Shijiazhuang No. 4 Pharmaceutical Co., Ltd.); $2 \%$ lidocaine hydrochloride injection (Shandong Hualu Pharmaceutical Co., Ltd.); epinephrine hydrochloride injection (Broad Pharmaceutical [China] Co., Ltd.); $10 \%$ glacial acetic acid (Taishan Xinning Pharmaceutical Co., Ltd.), absolute ethanol (Tianjin Yirenda Chemical Co., Ltd.), double-distilled water (Beijing Dongge Boye Biotechnology Co., Ltd.), PBS buffer (Beijing Dongge Boye Biotechnology Co., Ltd.); $10 \%$ neutral buffered formalin fixative (Beijing Yili Fine Chemical Co., Ltd.). Superoxide dismutase, reduced glutathione, malondialdehyde, advanced glycation end products, total collagen, collagen I, collagen III, elastin, and hyaluronic acid (HA) kits, provided by Beijing Dongge Boye Biotechnology Co., Ltd. (kit brand: Dogesce). Rabbit anti-CD31 polyclonal antibody (American Abcam Company), rabbit anti-VEGF polyclonal antibody (American Abcam Company), horseradish enzyme labeled goat anti-rabbit IgG (imported sub-packaging of Beijing Zhongshan Company), DAB color reagent kit (Beijing Zhongshan Biotechnology Co., Ltd.).

Main instruments: Medical purification workbench (CJ-2F, purification level 100, product of Suzhou Fengshi Experimental Animal Equipment Co., Ltd.); $-80{ }^{\circ} \mathrm{C}$ deep-low temperature refrigerator (Thermo Scientific Co. Ltd., U.S.A.), Medifuge CGF centrifuge (MF200, Silfradent, Italy); high-speed refrigerated centrifuge (Eppendorf AG, Germany); DHP-9162A electric-heating constant-temperature incubator (Guangzhou Shenhua Biotechnology Co., Ltd.); microplate reader (Rayto, RT-6100) (Shenzhen Rayto Life Science Co., Ltd.); DEM-3 plate washer (Beijing Tuopu Analytical Instrument Co., Ltd.); constant temperature shaker, model HT-111B (Shanghai Hetian 
Scientific Instrument Co., Ltd.); 10-100 $\mu$ l pipette (Eppendorf AG, Germany); automatic sample rapid grinder JXFSTPRP-32 (Shanghai Jingxin Technology Co., Ltd.); Synergy HT enzyme-linked immunosorbent assay detector (BioTek Company); electric thermostat (Chongqing Experimental Equipment Factory); paraffin slicer (Leica, Germany); paraffin-embedding machine (Leica, Germany); electronic constant-temperature water bath (Tianjin Taisite Test Instrument Co., Ltd.); electronic balance (Beijing Sartorius Balance Co., Ltd.); TGL-16B centrifuge (Shanghai Anting Scientific Instrument Factory); optical microscope (Olympus, Japan); automatic photomicrography system (Leica, German); low-temperature desktop ultracentrifuge (Eppendorf, Germany); pathology image analysis system (Clearing Imaging Company, Tongji Medical University).

\section{Experimental method}

Construction of D-galactose-induced aging model in nude mice: According to the experimental design, the backs of nude mice in the CGF group, $\mathrm{NF}$ group, CGF+NF group, and model group were subcutaneously injected with $1000 \mathrm{mg} / \mathrm{kg}$ D-half lactose. The $5 \%$ D-galactose solution was prepared by dissolving $5 \mathrm{~g}$ of D-galactose in $100 \mathrm{ml}$ of $0.9 \%$ sodium chloride injection. Once a day for eight weeks, before injection, the back skin of the nude mice was sprayed with $75 \%$ medical alcohol for disinfection.

Preparation of human CGF: After routine disinfection, a 9-ml green-tube-cap, negative-pressure special vacuum blood collection tube (containing heparin) was used to take a total of $18 \mathrm{ml}$ of the patient's cubital venous blood, and the two blood collection tubes were placed symmetrically in a centrifuge sterilized by ultraviolet light. The variable speed of centrifugation was set to 2,400-2,700 RPM, and the CGF was prepared by centrifugation according to the system setting program for 13 min. After centrifugation, a long 20G needle was placed on the top of the RBC to move the albuginea and carefully draw out about $2.5 \mathrm{ml}$ of the liquid containing the albuginea, which was CGF (about $0.5 \mathrm{ml}$ of the liquid is rich in the albuginea, that is, the CD34+ cell layer).

Preparation of human NF: Local anesthesia was used to enhance anesthesia (intravenous anesthesia), and about $300 \mathrm{ml}$ of swelling anesthetic solution was injected into the lower abdomen of healthy volunteers. According to the Coleman method, a 2-mm-outer-diameter porous blunt liposuction needle with a side hole $1 \mathrm{~mm}$ in diameter was used. A 20-ml spiral syringe with low negative pressure reciprocating liposuction was connected, and a total of about $133 \mathrm{ml}$ of the liquid lipid mixture was sucked out. The abdominal operation area was flat and without depression. After the mixture sits for $30 \mathrm{~min}$, the fat and the liquid below were stratified. After removing the water, the middle and lower fat were taken in the syringe, and two $10-\mathrm{ml}$ syringes were connected with "herringbone" (translated into a Mercedes-Benz shape abroad), "cross" (translated into a BMW shape abroad), and "five-star" emulsifiers in sequence. The injection was repeated 30 times to fully emulsify to prepare $10.8 \mathrm{ml}$ of NF ; then, the fibrous tissues were filtered out with stainless-steel filters with pore diameters of $1 \mathrm{~mm}$, $0.8 \mathrm{~mm}$, and $0.5 \mathrm{~mm}$, and the fat filtrate was NF.

Group intervention: After eight weeks of modeling, the corresponding preparations were injected subcutaneously and intracutaneously into the backs of nude mice in the CGF group, NF group, and CGF+NF group. All three groups were sterilized with $75 \%$ medical alcohol before injection. The CGF group was injected subcutaneously and intradermally with $0.5 \mathrm{ml}$ of CGF, the NF group was injected with $0.5 \mathrm{ml}$ of NF, and the CGF+NF group was injected with $0.25 \mathrm{ml}$ of CGF and $0.25 \mathrm{ml}$ of $\mathrm{NF}$ mixture. The normal control group (normal) and model group (model) did not undergo any treatment. After the injection, all nude mice were raised normally according to the original method.

Specimen collection: After 4 weeks of treatment, the serum and back skin specimens were collected from 5 groups of nude mice. The blood was about $0.5 \mathrm{ml}$. After sitting for a few minutes, it was placed in a high-speed centrifuge at 3000 RPM and centrifuged for five minutes. Then, $0.25 \mathrm{ml}$ of the upper layer of serum was transferred to the cryotube with a pipette and stored in the refrigerator at $-80{ }^{\circ} \mathrm{C}$ for later use. The back skin on both sides of the spine was cut about $1.5 \mathrm{~cm} \times 2 \mathrm{~cm}$. After sterile gauze was dipped into the skin blood, the left skin was put into $10 \%$ neutral buffered formalin solution for routine pathological examination and immunohistochemical examination. The right skin was put into a cryopreservation tube and immediately put into the refrigerator at $-80{ }^{\circ} \mathrm{C}$ for skin homogenate.

Measurement of serum and skin biochemical indexes: Skin samples stored in $-80^{\circ} \mathrm{C}$ refrigerator were taken out, $0.1 \mathrm{~g}$ of sample was added to a $0.9 \mathrm{ml}$ volume of physiological saline and the supernatant was obtained by centrifugal method and used for tested. The SOD was measured by the xanthine oxidase method, and the MDA 
was measured by the thiobarbituric acid method. The reduced glutathione (GSH), advanced glycation end products (AGEs), total collagen, collagen I, collagen III, elastin, and HA were measured by the ELISA method.

Histological observation: The back skin of nude mice was fixed with $10 \%$ neutral buffered formalin solution for $24 \mathrm{~h}$, and embedded in paraffin. Then, $4-\mu \mathrm{m}-$ thick paraffin sections were made and stained with $\mathrm{H} \& \mathrm{E}$, elastic fiber, and Masson.

Back skin thickness measurement of nude mice: The back skin tissue of each group of nude mice was taken from the $10 \%$ neutral buffered formalin solution and cut into thin slices. All skin tissue sections were stained with HE. The thickness of the dermis was measured at five locations of the same interval under the microscope with a ruler attached to the microscope, and the average thickness of the dermis was calculated. For each sample, the thickness of the dermis at five locations was measured independently, and the average was taken as the sample value. The thickness of the dermis of the skin tissues of each group was compared.

Skin collagen content distribution: Masson staining showed the skin collagen content, and the pathological image analysis system was used to measure the gray value of Masson staining in the superficial dermis of the skin, reflecting the content of collagen.

Changes in skin elastin content: Elastic fiber staining showed the skin elastin content, and the pathological image analysis system was used to measure the gray value of the elastin staining in the superficial dermis of the skin, reflecting the elastin content.

Determination of the number of dermal capillaries: The primary antibody CD31 was incubated at $4{ }^{\circ} \mathrm{C}$ overnight, and the catalase-carrying goat anti-mouse secondary antibody was incubated at $37^{\circ} \mathrm{C}$ for $30 \mathrm{~min}$. Then, DAB chromogenic fluid was used to develop the color under a light microscope. The CD31 immunohistochemical staining showed small blood vessels. Five fields of view were randomly selected for each slice under a 200-fold light microscope, and the number of microvessels in each photo was counted. The average value was taken as the number of microvessels in the sample.

Determination of VEGF expression in the superficial dermis: The primary antibody was VEGF, which was incubated overnight at $4{ }^{\circ} \mathrm{C}$. The catalasecarrying goat anti-mouse secondary antibody was incubated at $37{ }^{\circ} \mathrm{C}$ for $30 \mathrm{~min}$. Then, DAB chromogenic fluid was used to develop the color under a light microscope. The pathological image analysis system was used to determine the gray value of the VEGF immunohistochemical staining in the superficial dermis of the skin, reflecting the content of VEGF.

\section{Statistical analysis}

All the data obtained in this study were statistically analyzed by the SPSS 22.0 software. The experimental results of all measurement data were expressed as the mean \pm standard deviation $(x \pm s)$. It was used to judge whether the data obeyed the normal distribution and homogeneity of variance. A one-way analysis of variance or $t$-test was used for statistical processing, and $P<0.05$ indicated that the difference was statistically significant.

\section{Results}

The general condition and weight change of the five groups of nude mice

There was no significant difference in the body weights of the five groups of nude mice before the model was constructed. After eight weeks of modeling, the nude mice in the control group were quiet, full of energy, and quick to respond, and their skin was shiny and elastic; the nude mice of the skin aging model were easily frightened, and their skin was slightly dull and lost elasticity. The nude mice in the three treatment groups also showed susceptibility to fright but had better skin gloss.

Changes of SOD, MDA, and GSH levels in serum and skin

Compared with the normal group and the three treatment groups, the SOD of the model group was significantly lower $(P<0.05)$, and the SOD of the $\mathrm{NF}$ group was lower than that of the $\mathrm{CGF}+\mathrm{NF}$ group $(P=0.031$, Fig. 1A). Compared with the normal group, the serum MDA in the model group was significantly higher $(P<0.001)$. The MDA of the three treatment groups was significantly lower than that of the model group $(P<0.05)$, but the MDA of the CGF group $(P=0.01)$ and the NF group $(P=0.001)$ was still higher than that of the normal control group. There was no significant difference between the $\mathrm{CGF}+\mathrm{NF}$ group and the normal control group ( $P=0.409$, Fig. $1 \mathrm{~B})$. The serum $\mathrm{GSH}$ of the model group was significantly lower than that of the other groups $(P<0.05)$. The GSH of the NF group $(P=0.011)$ and the CGF group $(P=0.002)$ was significantly lower than that of the normal group. There was no significant 
difference between the $\mathrm{CGF}+\mathrm{NF}$ group and the normal group (Fig. 1C).

The skin SOD content of the nude mice in the model group and three treatment groups was lower than that in the normal group $(P<0.05)$, and the decline in the model group was the most obvious $(P<0.001)$. The SOD content of the three treatment groups was significantly higher than that of the model group $(P<0.05$, Fig. 2A). The skin MDA content of the nude mice in the model group, CGF group, and NF group was significantly higher than that in the control group $(P<0.05)$, and the MDA content of the $\mathrm{CGF}+\mathrm{NF}$ group was not different from that of the normal control group $(P=0.558)$. The MDA content of the CGF group, the NF group, and the $\mathrm{CGF}+\mathrm{NF}$ group was significantly lower than that of the model group $(P<0.05)$. The MDA content of the NF group and the CGF group was lower than that of the $\mathrm{CGF}+\mathrm{NF}$ group $(P<0.05$, Fig. 2B). The skin GSH content of the model group was significantly lower than that of other different treatment groups $(P<0.05)$. The GSH content of the CGF group, the NF group, and the $\mathrm{CGF}+\mathrm{NF}$ group was significantly higher than that of the model group, and there was no significant difference from that of the normal control group (Fig. 2C).
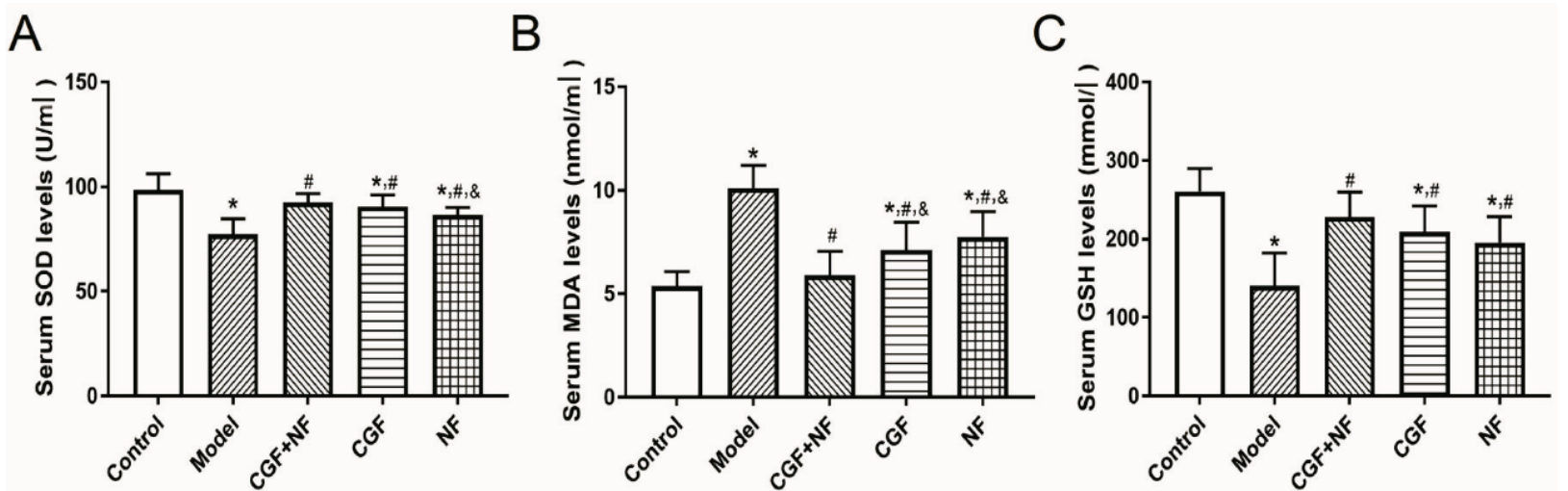

Fig. 1. The levels of oxidative stress markers in serum. (A) The levels of SOD in serum; (B) The levels of MDA in serum; (C) The levels of GSH in serum. ${ }^{*} P<0.05$ vs. control group; ${ }^{\#} P<0.05$ vs. model group; ${ }^{\&} P<0.05$ vs. CGF+NF group. SOD, superoxide dismutase; MDA, malondialdehyde; GSH, glutathione.
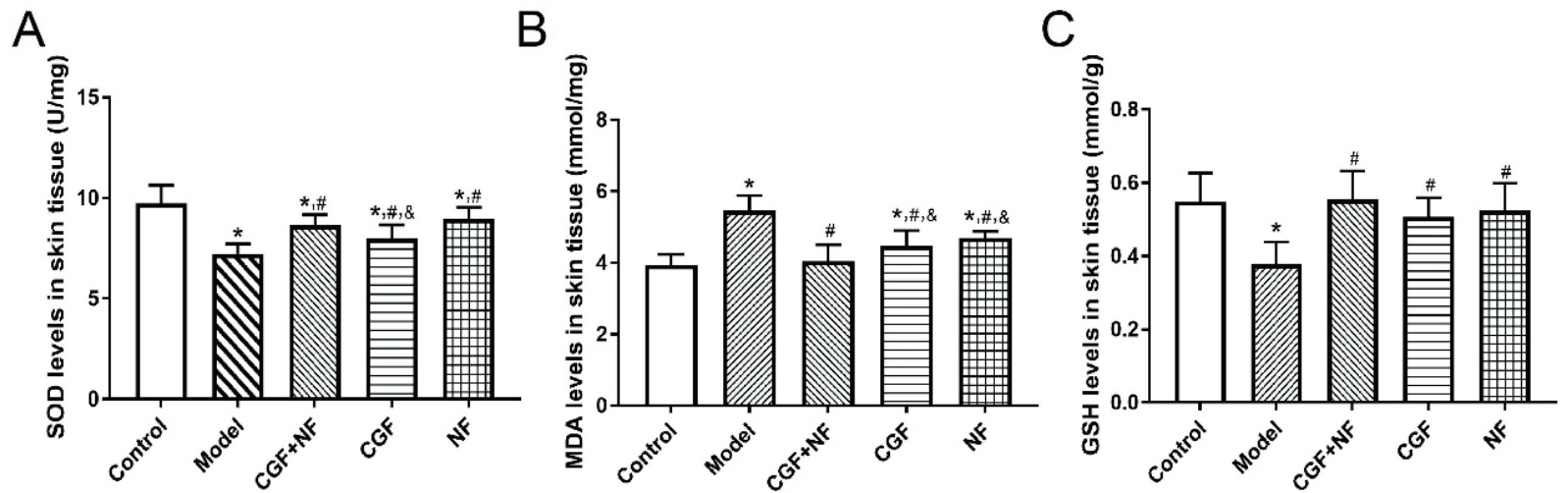

Fig. 2. The levels of oxidative stress markers in tissues. (A) The levels of SOD in tissues; (B) The levels of MDA in tissues; (C) The levels of GSH in tissues. $* P<0.05$ vs. control group; ${ }^{*} P<0.05$ vs. model group; ${ }^{\&} P<0.05$ vs. CGF+NF group. SOD, superoxide dismutase; MDA, malondialdehyde; GSH, glutathione.

Changes of total collagen, collagen I, collagen III, AGE, HA and elastin content in skin

Masson staining was used to show the distribution of skin collagen (Fig. 8A), and the gray value was used to determine the collagen content. The results showed that compared with the control group, the collagen fiber content of the model group was significantly decreased $(P<0.001)$. Compared with the model group, the collagen fibers in the three treatment groups were significantly increased $(P<0.05$, Fig. $8 \mathrm{~B})$. ELISA analysis also yielded the same result, as shown in Figure $3 \mathrm{~A}$, the total collagen content in the NF group and the model group decreased to varying degrees compared with that in normal control group $(P<0.05)$. The decrease 
in the model group was more significant, while the content in the CGF group and the mixed-treatment group did not decrease significantly $(P>0.05)$. Compared with the model group, the difference between the CGF group and the CGF+NF group was very significant $(P<0.05)$, and the total collagen content of the NF group was statistically different from that of the model group $(P<0.05)$. As shown in Figure 3B, compared with the normal control group, the collagen I content of the $\mathrm{NF}$ group and the model group decreased $(P<0.05)$, and the decrease was more obvious in the model group. The collagen I content in the three treatment groups was significantly higher than that in the model group $(P<0.05)$. As shown in Figure 3C, the collagen III content of the model group and the NF group was significantly lower than that of the normal group. The collagen III content in the three treatment groups was higher than that of the model group, especially in the CGF group and the mixed-treatment group $(P<0.05)$.
A

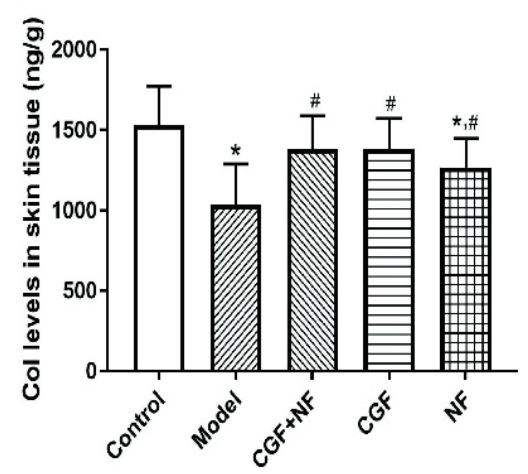

B

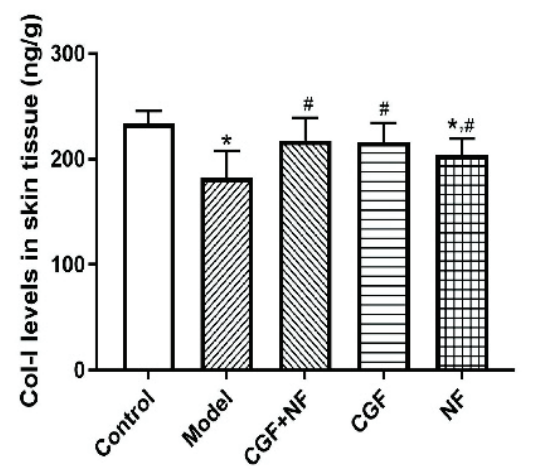

C

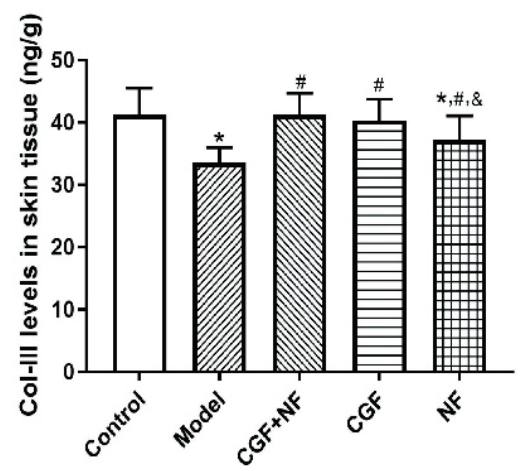

Fig. 3. The levels of collagen in skin tissues. (A) Col levels in skin tissues; (B) Col-I levels in skin tissues; (C) Col-III levels in skin tissues. * $P<0.05$ vs. control group; ${ }^{*} P<0.05$ vs. model group; ${ }^{\&} P<0.05$ vs. CGF+NF group. Col, collagen; Col-I, type I collagen; Col-III, type III collagen.

Compared with the normal control group, the skin AGE content of the nude mice in the model group and the NF group was significantly increased $(P<0.05)$; the skin AGE content of the CGF group and the $\mathrm{CGF}+\mathrm{NF}$ group was significantly lower than that of the model group $(P<0.05$, Fig. 4). The skin HA content of the nude mice in the model group was significantly lower than that of the mice in the other four groups $(P<0.05)$. Compared with the normal control group, the HA content of the three treatment groups did not change significantly, and there was no statistical difference between the three treatment groups (Fig. 6).

Compared with the normal control group, the skin elastin content of the nude mice in the model group and the NF group decreased significantly $(P<0.05)$, but there was no significant decrease in the CGF group and the $\mathrm{CGF}+\mathrm{NF}$ group. Compared with the model group, the elastin content of the CGF group and the $\mathrm{CGF}+\mathrm{NF}$ group was significantly higher. The elastin content of the NF group was slightly higher than that of the model group, but there was no statistical significance (Fig. 5). Meanwhile, elastic fiber staining was used to show the distribution of skin elastic fibers (Fig. 9A), and gray values were used to determine the collagen content. The results showed that compared with the control group, the elastic fiber content of the model group was significantly decreased $(P<0.05)$, and the elastic fiber of the three treatment groups was significantly increased $(P<0.05$, Fig. 9B).

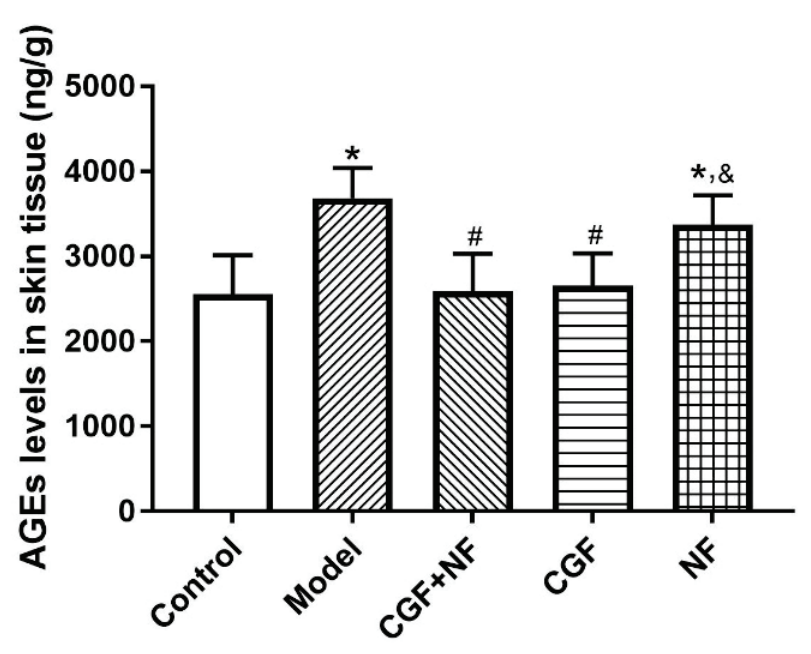

Fig. 4. The levels of AGEs in skin tissues. $* P<0.05$ vs. control group; ${ }^{\#} P<0.05$ vs. model group; ${ }^{\&} P<0.05$ vs. CGF+NF group. AGEs, advanced glycation end products. 


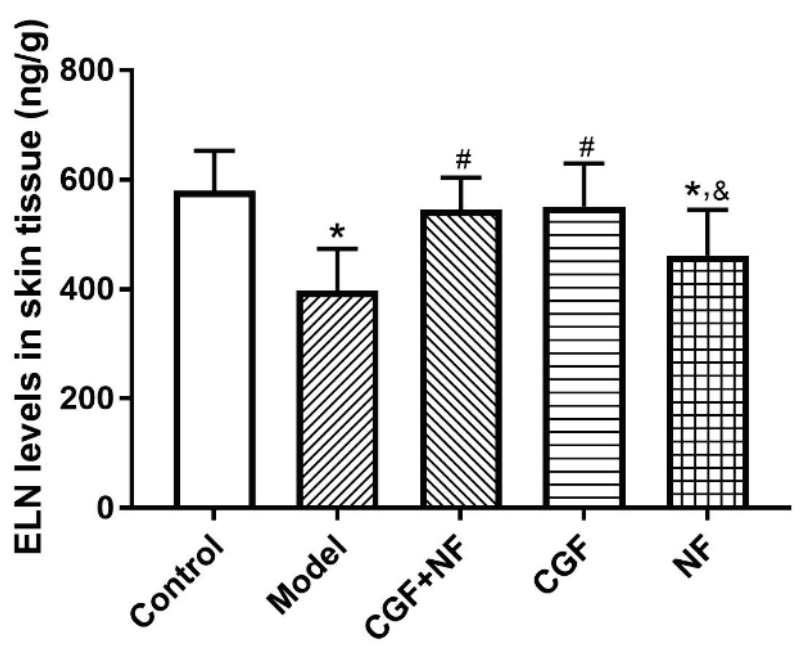

Fig. 5. The levels of ELN in skin tissues. $* P<0.05$ vs. control group; ${ }^{\#} P<0.05$ vs. model group; ${ }^{\&} P<0.05$ vs. CGF+NF group. ELN, elastin.

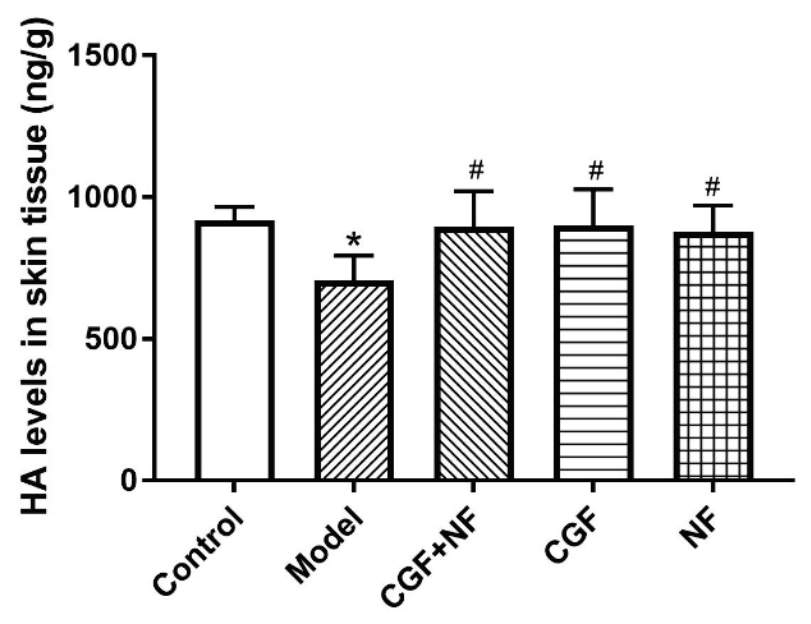

Fig. 6. The levels of $\mathrm{HA}$ in skin tissues. $* P<0.05$ vs. control group; ${ }^{\#} P<0.05$ vs. model group. HA, hyaluronic acid.

\section{The thickness of the dermis of nude mice}

The histological observation of the HE staining showed that the dermis of the nude mice turned red; the nucleus was dark blue, and a large number of dark blue spindle-shaped fibroblast nuclei could be seen in the collagen fibers (Fig. 7A). The dermis thickness of each skin sample was statistically analyzed under an optical microscope. Compared with the normal control group, the dermis of the nude mice in the model group was significantly thinner, and the dermis of the CGF group, the NF group, and the CGF+NF group was significantly thicker than that of the model group, which was statistically significant $(P<0.05$, Fig. 7B).

\section{Expression of CD31 and VEGF in skin}

The immunohistochemistry showed that compared with the control group, the number of CD31 vessels in the dermis in the model group was significantly reduced $(P<0.05)$, and the number of CD31 vessels in the dermis/HPF in the three treatment groups was significantly increased $(P<0.01)$. Compared with the model group, the number of CD31 dermal vessels/HPF in the treatment group was significantly increased $(P<0.01$, Fig. 10).

The immunohistochemistry showed that compared with the control group, the expression of VEGF in the dermis of the model group was significantly decreased $(\mathrm{P}<0.01)$, and the expression of VEGF in the treatment group was significantly increased $(P<0.01)$. Compared with the model group, the expression of VEGF in the treatment group increased significantly in the dermis $(P<0.01$, Fig. 11).

Note: ${ }^{*} P<0.05$ vs. control; ${ }^{\#} P<0.05$ vs. model group; ${ }^{\&} P<0.05$ vs. $\mathrm{CGF}+\mathrm{NF}$ group.
A

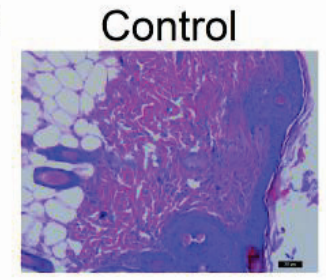

Model

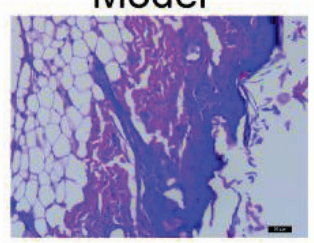

$\mathrm{CGF}+\mathrm{NF}$

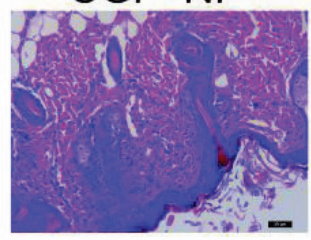

B

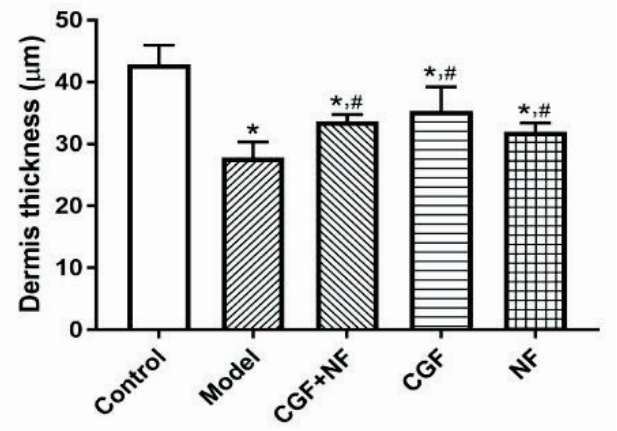

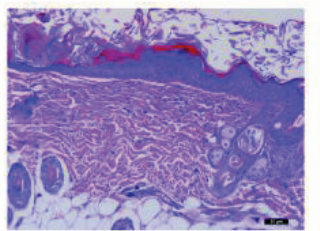

CGF

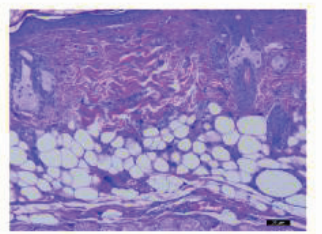

NF

Fig. 7. The dermis thickness in each group measured by H\&E staining. (A) The representative images of each group $(\times 100)$. (B) The comparison of dermis thickness in each group. ${ }^{*} P<0.05$ vs. control group; ${ }^{\#} P<0.05$ vs. model group. 

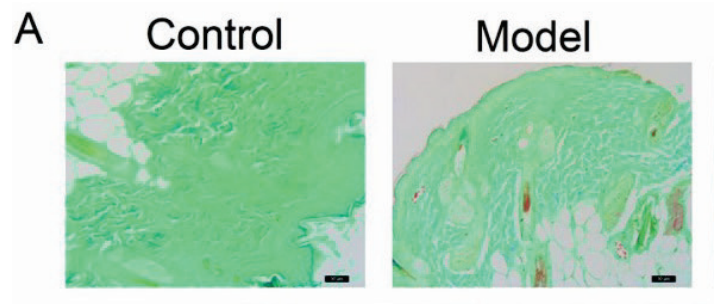

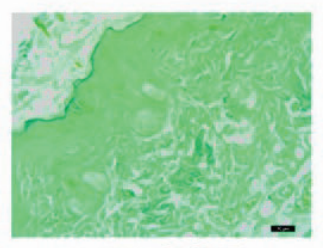

CGF

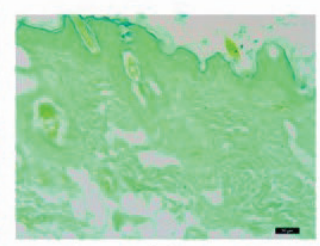

$\mathrm{NF}$
B

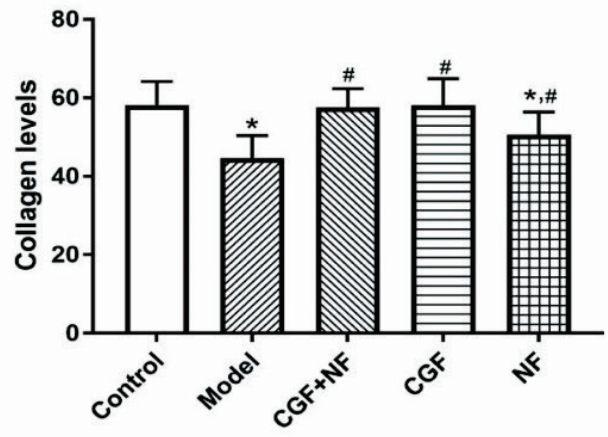

Fig. 8. The collagen levels in each group measured by Masson staining. (A) The representative images of each group $(\times 100)$. $(\mathbf{B})$ The comparison of collagen levels in each group. ${ }^{*} P<0.05$ vs. control group; ${ }^{\#} P<0.05$ vs. model group.
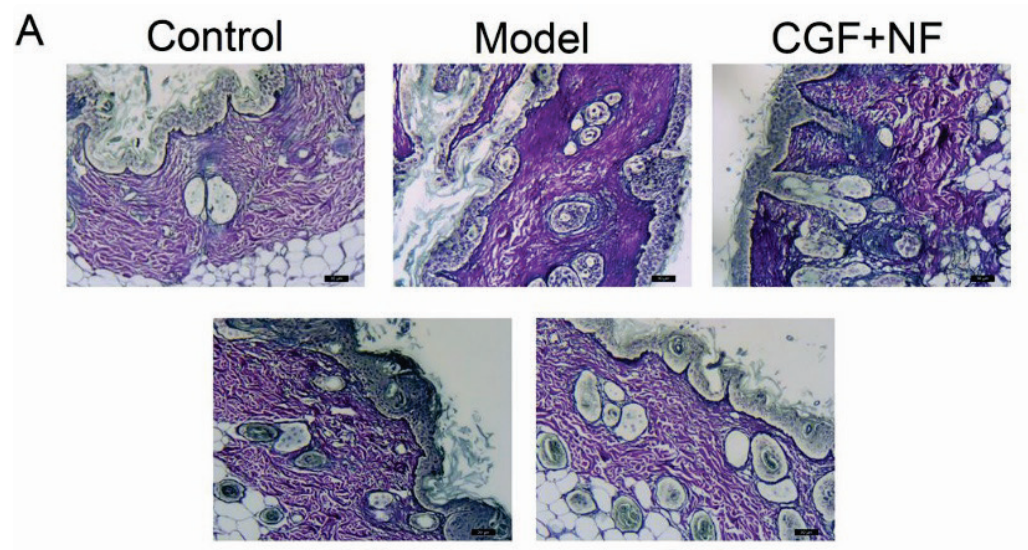

CGF

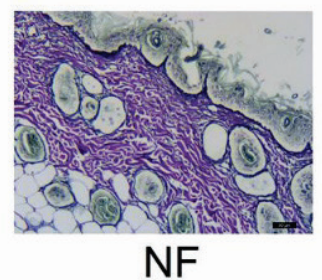

$B$

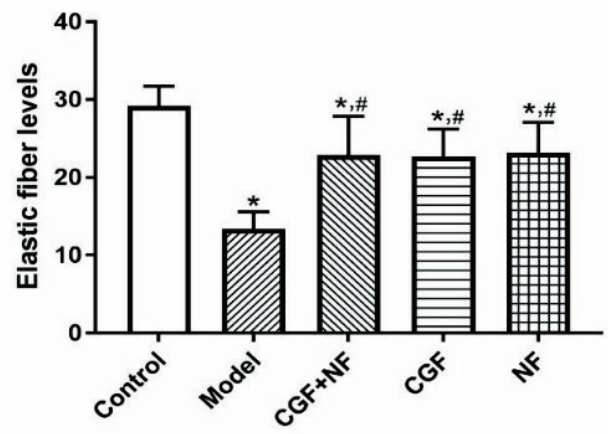

Fig. 9. The elastic fiber levels in each group. (A) The representative images of each group $(\times 100)$. (B) The comparison of elastic fiber levels in each group. ${ }^{*} P<0.05$ vs. control group; ${ }^{\#} P<0.05$ vs. model group.

\section{Discussion}

D-galactose is a reductive aldose that occurs naturally in the body and is normally metabolized to glucose. A large number of studies have shown that mice injected with D-galactose solution for a long time will produce excessive reactive oxygen species (ROS) in the body, and continuously enhanced oxidative stress will reduce the activity of antioxidant enzymes in various organs ( $\mathrm{Qu}$ et al. 2016, Gong et al. 2016). It causes damage to all organs of the body and decline in system functions, and the multi-tissue, multi-organ, and multilevel damage to the body conforms to the law of natural aging and shows physiological changes similar to natural aging (Hadzi-Petrushev et al. 2015). Due to the short modeling time, easy modeling, and good repeatability, this method has been widely used and has been reported in a large number of literature works (Song et al. 1999).
CGF is a third-generation plasma extract that seems to have better regeneration ability and versatility. The potential of this preparation lies in its fibrin network, containing platelets, white blood cells, growth factors, and $\mathrm{CD} 34+$ stem cells. It can provide a microenvironment for cell migration. The formation of new tissue generally requires two conditions: angiogenesis and collagen formation. At present, only CGF can achieve these two aspects. It is an autologous mesoderm therapy. It is injected into the dermis to induce the formation of collagen through its own blood cells, tighten the face, reduce dark lines, promote skin regeneration, and achieve facial rejuvenation. In 2013, Tonnard first proposed the concept of nanofat (Tonnard et al. 2013). In recent years, fat transplantation has been widely used in tissue regeneration and improving skin quality. Nanofat contains rich growth factors and adipogenic stem cells (ASCs), and has been clinically applied in the treatment 
of skin wrinkles and pigmentation (Lo Furno et al. 2017). Tonnard et al. injected nanofat into facial wrinkles, dark eye area, deep dermis and subdermis of cleavage skin wrinkles, and the wrinkles and dark eye circles were significantly improved, with good clinical effect (Tonnard et al. 2013). Kim and Lee found that the supernatant of ASCs can accelerate the proliferation of fibroblasts in the dermis and the secretion of collagen I and that it also has the ability to reduce the apoptosis of fibroblasts (Kim et al. 2009, Lee et al. 2009). In addition, the injection of ASCs can reduce mouse skin wrinkles caused by ultraviolet $\mathrm{B}$ and increase the content of collagen and the thickness of the dermis. Similar changes were also observed in this experiment. In this study, the results of the HE staining and Masson staining showed that the injection of D-galactose may cause thinning of the dermis of the skin and decrease the secretion of collagen. However, CGF, NF, and their mixture could reverse the changes in mouse dermis thickness and collagen secretion induced by D-galactose. The ELISA results showed that these ingredients could improve the secretion of mouse total collagen, collagen I, and collagen III induced by D-galactose.
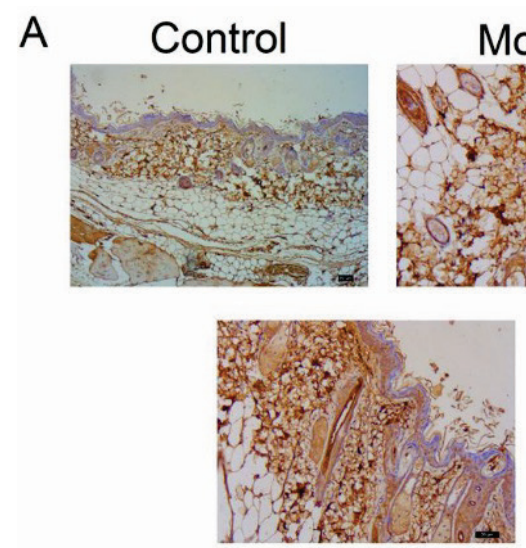

CGF
Model
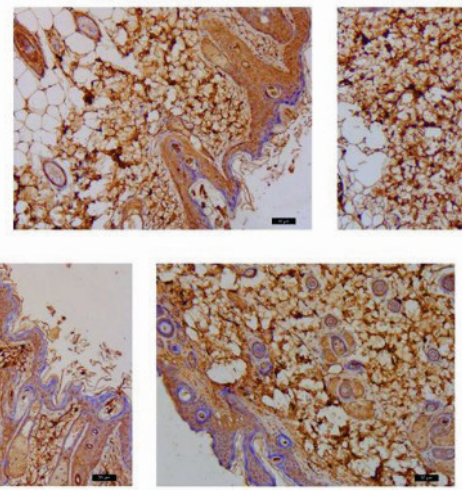

NF

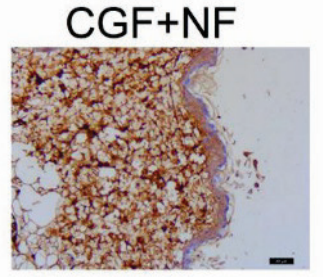

B

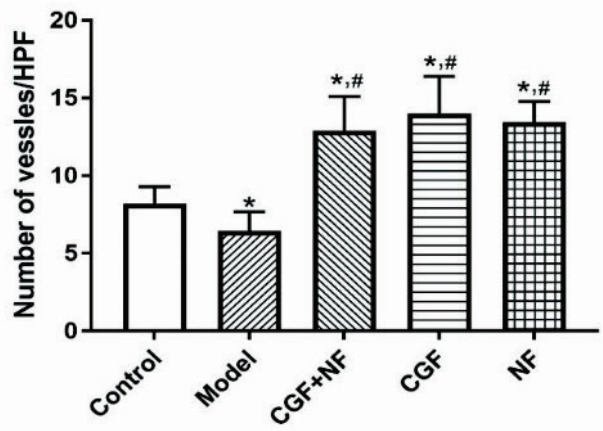

Fig. 10. The microvascular density in each group. (A) The representative images of each group $(\times 200)$. (B) The comparison of mean microvascular density in each group. $* P<0.05$ vs. control group; ${ }^{*} P<0.05$ vs. model group.

A
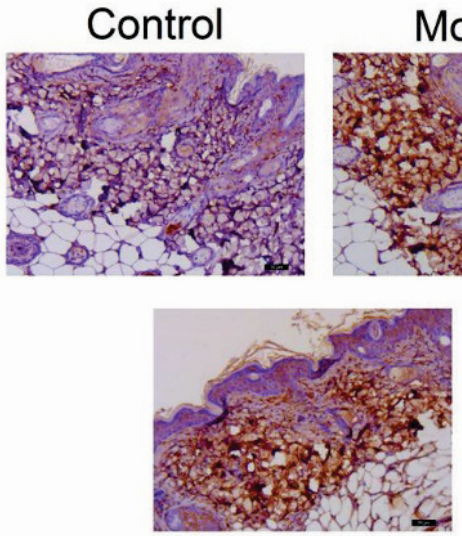

CGF
Model
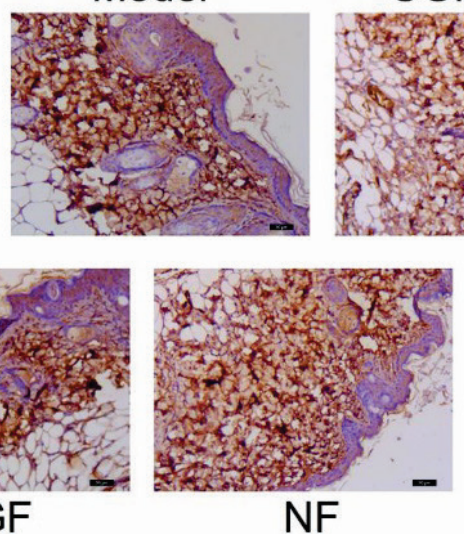

CGF+NF

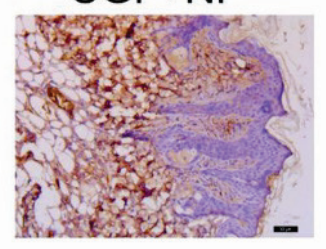

NF
B

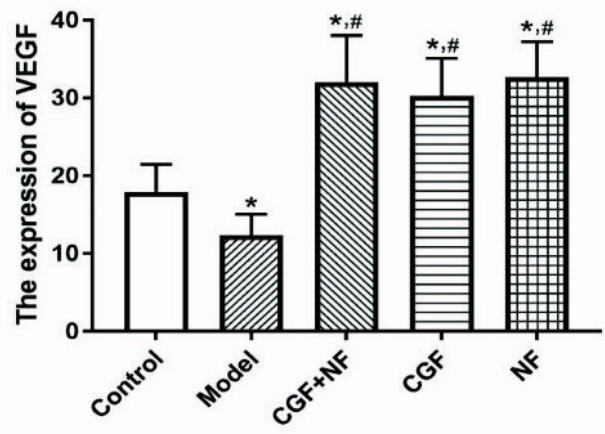

Fig. 11. The expression of VEGF in each group. (A) The representative images of each group $(\times 200)$. (B) The comparison of VEGF levels in each group. ${ }^{*} P<0.05$ vs. control group; ${ }^{*} P<0.05$ vs. model group. VEGF, vascular endothelial growth factor.

SOD and GSH peroxidase are important peroxidase-decomposing enzymes widely present in the body that can reflect the body's antioxidant capacity (Wang et al. 2013). The activity of SOD and the content of MDA have been used as two of the indicators that reflect the body's aging (Wu et al. 2018, Tsukahara et al. 2001). The latter can catalyze reduced GSH to oxidized glutathione, reduce toxic hydrogen peroxide to non-toxic 
hydroxyl compounds, eliminate excessive free radicals (ROS) in the body, and reduce the production of lipid peroxide MDA. Additionally, SOD and GSH can protect biological membranes from ROS damage and maintain normal cell function. With age, the activity of SOD and the content of GSH continue to decrease, which leads to an increase in the content of MDA, resulting in skin aging. However, subcutaneous injection of CGF and NF can slow down or reverse this phenomenon, thus showing their anti-aging effect. As expected, we found that the SOD and GSH content in skin and serum of the three treatment groups was significantly higher than that in model group, and the MDA content was significantly lower than that in model group. These results indicate that these components can effectively improve the activity of antioxidant enzymes in the skin, enhance the ability of skin tissue to scavenge free radicals and antioxidation, reduce skin oxidative damage, and thus play an anti-aging role. Meanwhile, the SOD and GSH content in the $\mathrm{NF}+\mathrm{CGF}$ combined treatment group was higher and the MDA content was lower, there was no significant difference from that of the normal control group. This shows the advantage of NF+CGF combined therapy.

Elastin plays a very important role in maintaining skin elasticity. Studies have shown that the increase of elastase secreted by aging fibroblasts leads to the decrease of elastic fibers and the curling of elastic fibers; as a result, the elasticity of skin decreases, the skin becomes loose, and wrinkles appear (Tsukahara et al. 2001). Our research finds that the injection of CGF, NF, or a mixture of both increased the content of skin tissue elastin and played an important role in fighting skin aging. It was speculated that this is related to the increase of skin elastic fiber synthesis or decrease of degradation after CGF or NF injection.

Due to the unique advantages and good application prospects of CGF and NF, many related basic and clinical studies have been done at home and abroad in recent years and achieved obvious results, but there are still the following problems to be solved: (1) There is no unified conclusion on the specific components and proportions of CGF and NF as mixtures. (2) There is a lack of systematic research on the time it takes the two to completely decompose in the body. (3) The interaction mechanism of the growth factors in them has not been fully elucidated. (4) There is a lack of long-term, largesample statistical results for the efficacy and safety of clinical applications. (5) Most clinical research uses subjective methods for evaluation, but for objective evaluation, methods such as CT, MRI, or soft-tissue three-dimensional photography could also be introduced (Herold et al. 2013). (6) There is still a lack of highquality clinical randomized controlled studies, and relevant studies are urgently needed to establish guidelines for the application of platelet concentrate products.

In conclusion, our study provides evidence that concentrated growth factor and nanofat can enhance the content of SOD and GSH in serum and skin of mice, reduce the level of MDA, significantly improve the content of collagen and elastin in skin, and have antiaging effect on skin. At the same time, the combined treatment of $\mathrm{CGF}+\mathrm{NF}$ has a better effect on alleviating skin aging and provides a new idea for the treatment of skin aging

\section{Conflict of Interest}

There is no conflict of interest.

\section{References}

DENG D, WANG W, WANG B, ZHANG P, ZHOU G, ZHANG WJ, CAO Y, LIU W: Repair of Achilles tendon defect with autologous ASCs engineered tendon in a rabbit model. Biomaterials 35: 8801-8809, 2014. https://doi.org/10.1016/j.biomaterials.2014.06.058

GONG YS, GUO J, HU K, GAO YQ, XIE BJ, SUN ZD, YANG EN, HOU FL: Ameliorative effect of lotus seedpod proanthocyanidins on cognitive impairment and brain aging induced by D-galactose. Exp Gerontol 74: 21-28, 2016. https://doi.org/10.1016/j.exger.2015.11.020

HA TS, SONG CJ, LEE JH: Effects of advanced glycosylation endproducts on perlecan core protein of glomerular epithelium. Pediatr Nephrol 19: 1219-1224, 2004. https://doi.org/10.1007/s00467-004-1590-1

HADZI-PETRUSHEV N, STOJKOVSKI V, MITROV D, MLADENOV M: D-galactose induced changes in enzymatic antioxidant status in rats of different ages. Physiol Res 64: 61-70, 2015. https://doi.org/10.33549/physiolres.932786

HEROLD C, UEBERREITER K, BUSCHE MN, VOGT PM: Autologous fat transplantation: volumetric tools for estimation of volume survival. A systematic review. Aesthetic Plast Surg 37: 380-387, 2013. https://doi.org/10.1007/s00266012-0046-4 
HERRLING T, JUNG K, FUCHS J: The role of melanin as protector against free radicals in skin and its role as free radical indicator in hair. Spectrochim Acta A Mol Biomol Spectrosc 69: 1429-1435, 2008. https://doi.org/10.1016/j.saa.2007.09.030

KIM WS, PARK BS, SUNG JH: The wound-healing and antioxidant effects of adipose-derived stem cells. Expert Opin Biol Ther 9: 879-887, 2009. https://doi.org/10.1517/14712590903039684

LEE EY, XIA Y, KIM WS, KIM MH, KIM TH, KIM KJ, PARK BS, SUNG JH: Hypoxia-enhanced wound-healing function of adipose-derived stem cells: increase in stem cell proliferation and up-regulation of VEGF and bFGF. Wound Repair Regen 17: 540-547, 2009. https://doi.org/10.1111/j.1524-475X.2009.00499.x

LIU JX, JIANG XM, SHEN J, ZHANG H: Chemical properties of superfine pulverized coal particles. Part 1. Electron paramagnetic resonance analysis of free radical characteristics. Adv Powder Technol 25: 916-925, 2014. https://doi.org/10.1016/j.apt.2014.01.021

LO FURNO D, TAMBURINO S, MANNINO G, GILI E, LOMBARDO G, TARICO MS, VANCHERI C, GIUFFRIDA R, PERROTTA RE: Nanofat 2.0: experimental evidence for a fat grafting rich in mesenchymal stem cells. Physiol Res 66: 663-671, 2017. https://doi.org/10.33549/physiolres.933451

OSAWA M, HANADA K, HAMADA H, NAKAUCHI H: Long-term lymphohematopoietic reconstitution by a single CD34-low/negative hematopoietic stem cell. Science 273: 242-524, 1996. https://doi.org/10.1126/science.273.5272.242

QU Z, ZHANG J, YANG H, HUO L, GAO J, CHEN H, GAO W: Protective effect of tetrahydropalmatine against D-galactose induced memory impairment in rat. Physiol Behav 154: 114-125, 2016. https://doi.org/10.1016/i.physbeh.2015.11.016

SONG X, BAO M, LI D, LI YM: Advanced glycation in D-galactose induced mouse aging model. Mech Ageing Dev 108: 239-251, 1999. https://doi.org/10.1016/S0047-6374(99)00022-6

TONNARD P, VERPAELE A, PEETERS G, HAMDI M, CORNELISSEN M, DECLERCQ H: Nanofat grafting: basic research and clinical applications. Plast Reconstr Surg 132: 1017-1026, 2013. https://doi.org/10.1097/PRS.0b013e31829fe1b0

TSUKAHARA K, TAKEMA Y, MORIWAKI S, TSUJI N, SUZUKI Y, FUJIMURA T, IMOKAWA G: Selective inhibition of skin fibroblast elastase elicits a concentration-dependent prevention of ultraviolet B-induced wrinkle formation. J Invest Dermatol 117: 671-677, 2001. https://doi.org/10.1046/j.0022-202x.2001.01450.x

WANG Z, LU CR, LUO Y, ET AL.: Evaluation of aging effect induced by D-galactose injection in rats. China Med Herald 10: 25-26, 2013.

WU YN, YIN XC, LI ZM, ET AL: Study on aging characteristics and aging markers of D-galactose aging model. Trop Med China 18: 682-686, 2018. 